\title{
Bioremediation and Phytoremediation of Soils Polluted With Hydrocarbons: Assumptions and Comments
}

\author{
Iván Balderas-León \\ Universidad Michoacana de San Nicolas de Hidalgo, México \\ E-mail: ivanguitarplayer@hotmail.com
}

José Luis Rico Cerda

Universidad Michoacana de San Nicolás de Hidalgo, México

E-mail: jlrico@umich.mx

Gustavo Santoyo Pizano

Universidad Michoacana de San Nicolás de Hidalgo, México

E-mail: gsantoyo@umich.mx

Liliana Márquez-Benavides

Universidad Michoacana de San Nicolas de Hidalgo, México

E-mail:1marquez@umich.mx

Blanca Celeste Saucedo-Martinez

Universidad Michoacana de San Nicolas de Hidalgo, México

E-mail: celsm@live.com.mx

Juan Manuel Sánchez-Yañez

Universidad Michoacana de San Nicolás de Hidalgo, México

E-mail: syanez@umich.mx 
Received: June 2, 2018 Accepted: October 4, 2018 Published: October 6, 2018

Doi: 10.5296/jab.v7i1.13243 URL: http://doi.org/10.5296/jab.v7i1.13243

\begin{abstract}
The studies reported in the literature dealing with remediation of soils polluted with hydrocarbons (HCs) very often conclude that the soil was remediated by following some specific actions, microorganisms, plants or both. However, when affirming this, attention should be taken, since environmental regulations related to HCs as pollutants commonly differ from one country to another. In addition, some other research reports on remediation lack of a local environmental regulation, which could indicate the concentration of HCs, permitted in soils. The present short review highlights the importance of these concepts.
\end{abstract}

Keywords: hydrocarbon, environmental regulation, bioremediation, phytoremediation

\title{
1. Introduction
}

According to national or international environmental regulations, a soil is contaminated with hydrocarbons (HCs) if the concentration is above a specific value (Kumar et al., 2011; Sharma, 2012). The level of HCs in impacted soil is due to anthropogenic reasons and the pollutants are commonly present as solids or liquids (Thapa et al., 2012; Nwoko, 2010; Haritash \& Kaushik, 2009; Johnsen et al., 2005). Depending upon the nature and concentration of HCs spilled on soil or in water, physical separation is sometimes possible, which include washing, encapsulating and vitrification, followed by chemical processes, such as immobilization, precipitation and oxidation. The remaining HCs are finally bioremediated or phytoremediated, or both (Juhasz \& Naidu, 2000; Gerhardt et al., 2009). The latter stage could be left to nature, or intentionally promoted by applying some specific microorganisms or plants, or both, able to eliminate the pollutants. Research reports in the literature apply bioremediation (BIO) or/and phytoremediation (PHYTO) to soils impacted with relative high concentrations of $\mathrm{HCs}$; however, the maximum concentration of $\mathrm{HCs}$, where bio or phytoremediation should be applied, is undefined. Furthermore, the lack of an environmental reference to indicate that the soil was remediated generates doubts about the results. And to add complexity, many countries have different environmental regulations related to specific HCs. This contribution selectively analyzes some papers to demonstrate the problem and highlights the urgency to have common environmental regulations when dealing with pollution of the environment.

\section{Assumptions and Comments}

Some research studies related to pollution of soils with HCs treated with BIO or PHYTO and use a vegetal and plant growth promoting microorganisms (PGPM) to solve the problem. However, this strategy could be unsuitable when the vegetal is not well selected and/or when the level of contamination is still far above the maximum permitted by the local environmental regulation. The Mexican environmental regulation (NOM-138-SEMARNAT/SS, 2012) allows the presence of $4400 \mathrm{ppm}$ of HCs in soils. But specifically, only $200 \mathrm{ppm}$ of light, $1200 \mathrm{ppm}$ of medium and $3000 \mathrm{ppm}$ of heavy fraction are 
permitted. Above $4400 \mathrm{ppm}$ of HCs the soil is considered as polluted and remediation is required. Table 1 presents some assumptions commonly found in research papers related to soils contaminated with HCs.

Table 1. Common assumptions reported in studies related to bioremediation or/and phytoremediation of soils polluted with hydrocarbons

\begin{tabular}{|c|c|}
\hline Assumptions & Comments \\
\hline $\begin{array}{l}\text { Contamination was remediated by a } \\
\text { biological action. }\end{array}$ & $\begin{array}{l}\text { It might not be the case, since biological } \\
\text { remediation is a function of the nature and } \\
\text { concentration of pollutant. }\end{array}$ \\
\hline $\begin{array}{l}\text { The environmental problem was } \\
\text { remediated by physical or mechanical } \\
\text { actions. }\end{array}$ & $\begin{array}{l}\text { It is usually untrue, since the concentration of } \\
\text { contaminant could still be severe. }\end{array}$ \\
\hline $\begin{array}{l}\text { The application of qualitative and } \\
\text { quantitative methods could indicate that } \\
\text { the soil was remediated. }\end{array}$ & $\begin{array}{l}\text { To assure that the problem was solved, The use } \\
\text { of bio-indicators is highly recommended. }\end{array}$ \\
\hline $\begin{array}{l}\text { The soil was remediated since the analysis } \\
\text { fulfills the requirements of a local } \\
\text { environmental regulation. }\end{array}$ & $\begin{array}{l}\text { All countries should be subjected to general } \\
\text { international environmental regulations. }\end{array}$ \\
\hline
\end{tabular}

Table 2 shows some selected studies where remediation of soil was assumed but the final HCs concentration in the soil was above $4400 \mathrm{ppm}$, the level permitted by the Mexican regulation (NOM-138-SEMARNAT/SS, 2012). In addition, even if the contamination level was below $4400 \mathrm{ppm}$, it could be treated as contaminated soil by other more severe international regulations. Table 2 also shows the lack of common criteria to consider that the soil was remediated. It is worth mentioning that the US Environmental Protection Agency for contamination of soil with HCs (Ohio EPA, 2010) indicates a maximum allowable concentration for each compound separately, instead of grouping the HCs as the NOM-138-SEMARNAT/SSA1-2012. It is worth commenting that the spills of HCs to the environment commonly occur as mixtures of HCs instead of a single compound. In addition, since fossil fuels strongly support the development of all societies in the world, proper remediation of the environment contaminated with HCs constitute a great challenge. 


\section{Macrothink}

Journal of Applied Biotechnology

ISSN 2327-0640 2019, Vol. 7, No. 1

Table 2. Some research studies related to soils contaminated with hydrocarbons which concluded that the soil was remediated. The final concentration reported was above 4400 ppm*, and a bio-indicator test was not performed

\begin{tabular}{|c|c|c|c|c|c|}
\hline Pollutant & $\begin{array}{l}\text { Initial } \\
\text { concentrati } \\
\text { on } \\
(\mathrm{ppm})\end{array}$ & $\begin{array}{l}\text { Final } \\
\text { concentrati } \\
\text { on }(\mathrm{ppm})\end{array}$ & Treatment & $\begin{array}{l}\text { Duratio } \\
\mathrm{n} \\
\text { (days) }\end{array}$ & Reference \\
\hline Petroleum & 30,000 & 17,700 & $\begin{array}{l}\text { Biostimulation } \\
\text { (BIS)-mineral } \\
\text { fertilization (MIFE) }\end{array}$ & 28 & $\begin{array}{l}\text { (Vallejo at al., } \\
2005)\end{array}$ \\
\hline $\begin{array}{l}\text { Used } \\
\text { motor oil }\end{array}$ & 40,000 & 12,320 & BIS-MIFE & 42 & $\begin{array}{l}\text { (Abdulsalam and } \\
\text { Omale 2009) }\end{array}$ \\
\hline Petroleum & 30,000 & 15,300 & $\begin{array}{l}\text { BIS- fertilization } \\
(\mathrm{FE})\end{array}$ & 195 & $\begin{array}{l}\text { (Asquith et al., } \\
\text { 2012) }\end{array}$ \\
\hline Petroleum & 50,000 & 31,000 & $\begin{array}{l}\text { Phytoremediaton } \\
\text { (PHYTO) } \\
\text { Brachiaria } \\
\text { brizantha }\end{array}$ & 120 & $\begin{array}{l}\text { (Merkl et al., } \\
2005)\end{array}$ \\
\hline Petroleum & 40,000 & 9,500 & $\begin{array}{l}\text { PHYTO-Sorghum } \\
\text { vulgare }\end{array}$ & 90 & $\begin{array}{l}\text { (Shirdam et al., } \\
\text { 2008) }\end{array}$ \\
\hline \multirow[t]{3}{*}{ Petroleum } & 23,000 & 16,100 & PHYTO-Vicia faba & 90 & (Diab, 2008) \\
\hline & & 19,130 & PHYTO-Zea mays & 90 & \\
\hline & & 19,850 & $\begin{array}{l}\text { PHYTO-Triticum } \\
\text { aestivum }\end{array}$ & & \\
\hline Petroleum & 75,000 & 44,000 & $\begin{array}{l}\text { PHTYO-Glycine } \\
\max \end{array}$ & 105 & $\begin{array}{l}\text { (Njoku \& } \\
\text { Akinola, 2009) }\end{array}$ \\
\hline Petroleum & 50,000 & 6,000 & $\begin{array}{l}\text { PHYTO-Vigna } \\
\text { unguiculata }\end{array}$ & 60 & $\begin{array}{l}\text { (Tanee and } \\
\text { Akonye, 2009) }\end{array}$ \\
\hline $\begin{array}{l}\text { Residual } \\
\text { motor oil }\end{array}$ & 25,000 & 8,175 & $\begin{array}{l}\text { PHYTO-Jatropha } \\
\text { curcas }\end{array}$ & 180 & $\begin{array}{l}\text { (Agamuth et al., } \\
\text { 2010) }\end{array}$ \\
\hline Petroleum & 40,000 & 17,920 & PHYTO-Zea mays & 120 & $\begin{array}{l}\text { (Zand el al., } \\
\text { 2010) }\end{array}$ \\
\hline Petroleum & 45,000 & 32,000 & $\begin{array}{l}\text { PHYTO-Zea mays } \\
\text { PHYTO-Avena } \\
\text { sativa }\end{array}$ & 30 & $\begin{array}{l}\text { (Merkl et al., } \\
2005)\end{array}$ \\
\hline Light oil & 81,500 & 49,878 & $\begin{array}{l}\text { PHYTO-Cyperus } \\
\text { rotundus }\end{array}$ & 180 & $\begin{array}{l}\text { (Basumatary et } \\
\text { al., 2012) }\end{array}$ \\
\hline
\end{tabular}




\begin{tabular}{|c|c|c|c|c|c|}
\hline Petroleum & 75,000 & 27,000 & $\begin{array}{l}\text { PHYTO-Sorghum } \\
\text { bicolor }\end{array}$ & 90 & $\begin{array}{l}\text { (Asiabadi et al., } \\
\text { 2014) }\end{array}$ \\
\hline \multirow[t]{2}{*}{ Diesel } & \multirow[t]{2}{*}{50,000} & 25,000 & $\begin{array}{l}\text { PHYTO-Lolium } \\
\text { perenne }\end{array}$ & 90 & \multirow{2}{*}{$\begin{array}{l}\text { (Chuluun et al., } \\
\text { 2014) }\end{array}$} \\
\hline & & 21,300 & $\begin{array}{l}\text { PHYTO-Lolium } \\
\text { perenne+BA }\end{array}$ & 90 & \\
\hline Petroleum & 50,000 & 25,700 & $\begin{array}{l}\text { PHYTO- Festuca } \\
\text { arundinacea }\end{array}$ & 150 & $\begin{array}{l}\text { (Tang et al., } \\
2010)\end{array}$ \\
\hline Diesel & 8,786 & 4,501 & $\begin{array}{l}\text { PHYTO- Zinnia } \\
\text { elegans }\end{array}$ & 180 & $\begin{array}{l}\text { (Ozawa et al., } \\
2015)\end{array}$ \\
\hline $\begin{array}{l}\text { Light } \\
\text { crude oil }\end{array}$ & 100,000 & 90,000 & $\begin{array}{l}\text { PHYTO- Sorghum } \\
\text { bicolor }\end{array}$ & 45 & $\begin{array}{l}\text { (Minai-Tehrani et } \\
\text { al., 2012) }\end{array}$ \\
\hline Gasoline & 30,000 & 5,010 & $\begin{array}{l}\text { PHYTO- Scirpus } \\
\text { mucronatus } \\
\text { inoculated with }\end{array}$ & 72 & $\begin{array}{l}\text { (Almansoory et } \\
\text { al., 2014) }\end{array}$ \\
\hline & & & Serratia marcescens & & \\
\hline \multirow{2}{*}{$\begin{array}{l}\text { Residual } \\
\text { lubricating } \\
\text { oil }\end{array}$} & 25,000 & 8,475 & $\begin{array}{l}\text { BIS-organic } \\
\text { wate-mushroom }\end{array}$ & 90 & \multirow{2}{*}{$\begin{array}{l}\text { (Abioye et al., } \\
2012 \text { ) }\end{array}$} \\
\hline & 10,000 & 8,225 & $\begin{array}{l}\text { compost- } \\
\text { Hibiscus cannbinus) }\end{array}$ & & \\
\hline \multirow{3}{*}{ Petroleum } & \multirow{3}{*}{60,600} & 32,118 & $\begin{array}{l}\text { Bioaugmetation } \\
\text { (BA)- Rhizopus sp }\end{array}$ & 35 & \multirow{3}{*}{$\begin{array}{l}\text { (Mancera-López } \\
\text { et al., 2008) }\end{array}$} \\
\hline & & 36,360 & $\begin{array}{l}\text { BA- Penicillium } \\
\text { funiculosum }\end{array}$ & & \\
\hline & & 33,330 & $\begin{array}{l}\text { BA-Aspergillus } \\
\text { sydowii }\end{array}$ & & \\
\hline $\begin{array}{l}\text { Oily-Slud } \\
\text { ge }\end{array}$ & 69,700 & 5,530 & $\begin{array}{l}\text { BA-BIS-Acinetobac } \\
\text { ter baumannii y } \\
\text { Burkholderia } \\
\text { cepacia, and } \\
\text { nutrients }\end{array}$ & 360 & $\begin{array}{l}\text { (Mishra et al., } \\
\text { 2001) }\end{array}$ \\
\hline Petroleum & 20,000 & 10,076 & $\begin{array}{l}\text { PHYTO Mirabilis } \\
\text { jalapa }\end{array}$ & 127 & $\begin{array}{l}\text { (Peng et al., } \\
2009)\end{array}$ \\
\hline Petroleum & 75,000 & 4,500 & $\begin{array}{l}\text { PHYTO } \\
\text { Sebastiania }\end{array}$ & 424 & $\begin{array}{l}\text { (Toledo Ramos } \\
\text { et al., 2009) }\end{array}$ \\
\hline Petroleum & 40,000 & 32,748 & $\begin{array}{l}\text { PHTYO Impatiens } \\
\text { balsamina }\end{array}$ & 120 & (Cai et al., 2010) \\
\hline Petroleum & 40,000 & 28,948 & Pharbitis & 127 & (Zhang \\
\hline
\end{tabular}




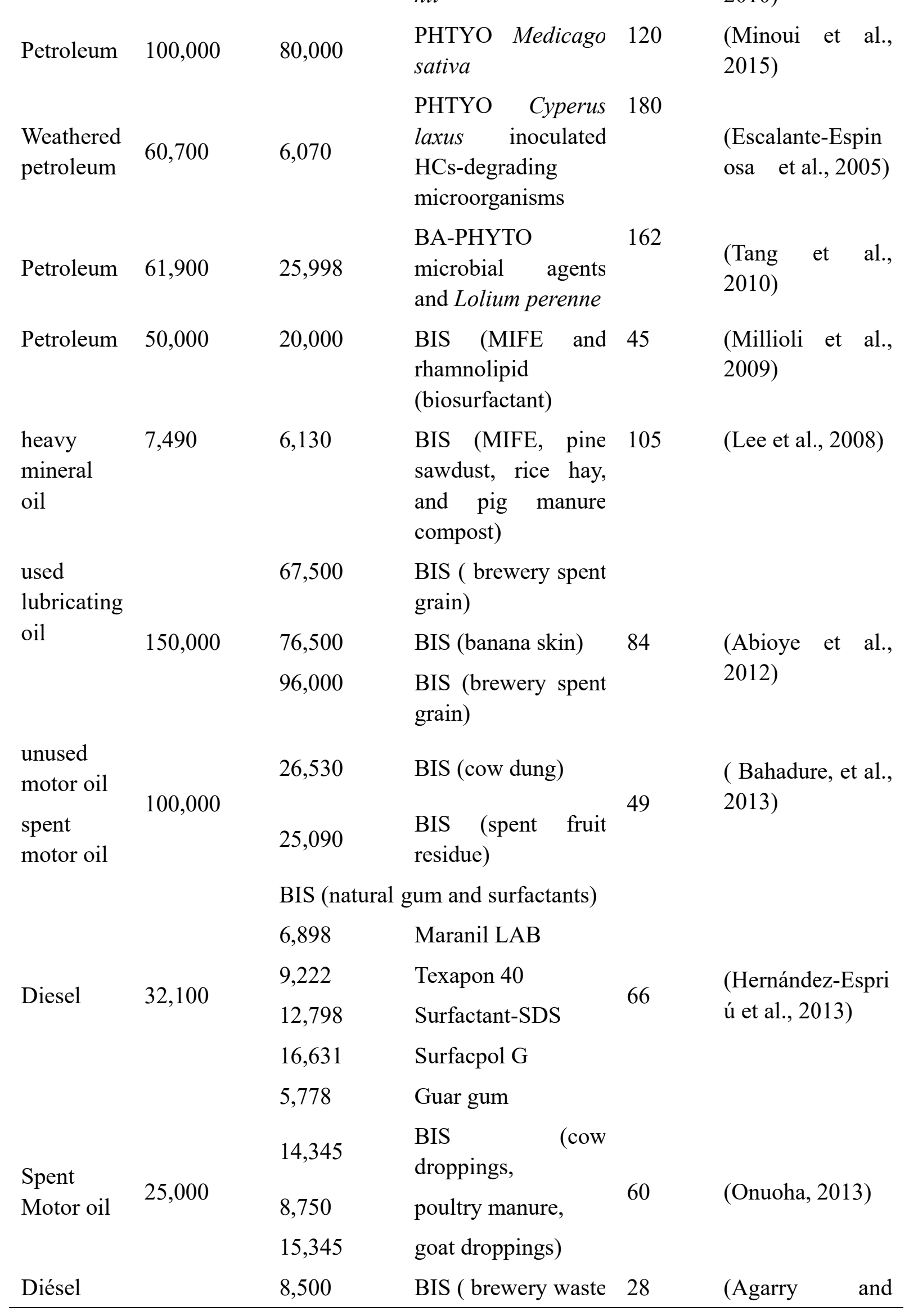




\begin{tabular}{|c|c|c|c|c|c|}
\hline & & & $\begin{array}{l}\text { effluents } \\
\text { bioventing) }\end{array}$ & & Latinwo 2015) \\
\hline \multirow{4}{*}{ Crude oil } & 100.000 & 5750 & BIS (plantain peels & \multirow{4}{*}{56} & \multirow{4}{*}{$\begin{array}{l}\text { (Romanus et al., } \\
\text { 2015) }\end{array}$} \\
\hline & & 4795 & & & \\
\hline & 150000 & 7260 & \multirow{2}{*}{ guinea corn shaf) } & & \\
\hline & 150,000 & 7350 & & & \\
\hline Gasoil & 40,000 & 8,800 & $\begin{array}{l}\text { BIS (MIFE and BA } \\
\text { by two indigenous } \\
\text { bacteria) }\end{array}$ & 45 & $\begin{array}{l}\text { (Najirad et al., } \\
\text { 2012) }\end{array}$ \\
\hline Crude oil & 52,000 & 10,600 & $\begin{array}{l}\text { BIS (MIFE, saw } \\
\text { dust, cow and sheep } \\
\text { dung and BA (by } \\
\text { specialized } \\
\text { microbial } \\
\text { consortium) }\end{array}$ & 90 & $\begin{array}{l}\text { (Burghal et al., } \\
\text { 2015) }\end{array}$ \\
\hline Crude oil & 170,000 & 65,000 & $\begin{array}{l}\text { BA (by biological } \\
\text { product: Amnite } \\
\text { P300 (Pseudomonas } \\
\text { Putida and Bacilli } \\
\text { Subtilis) }\end{array}$ & 118 & $\begin{array}{l}\text { (Benyahia et al., } \\
\text { 2005) }\end{array}$ \\
\hline \multirow{2}{*}{$\begin{array}{l}\text { Mineral } \\
\text { oil }\end{array}$} & \multirow{2}{*}{35,026} & & $\begin{array}{l}\text { Acinetobacter } \\
\text { calcoaceticus }\end{array}$ & \multirow{2}{*}{120} & \multirow{2}{*}{$\begin{array}{l}\text { (Aytkeldiyeva et } \\
\text { al., 2008) }\end{array}$} \\
\hline & & 6,966 & $\begin{array}{l}\text { Microbacterium } \\
\text { lacticum) }\end{array}$ & & \\
\hline \multirow{3}{*}{$\begin{array}{l}\text { Spent } \\
\text { diesel oil }\end{array}$} & 50,000 & 7,795 & \multirow{3}{*}{$\begin{array}{l}\text { BA (by Pleurotus } \\
\text { pulmonarius) }\end{array}$} & \multirow{3}{*}{60} & \multirow{3}{*}{$\begin{array}{l}\text { (Adenipeku, } \\
\text { 2008) }\end{array}$} \\
\hline & 10,000 & 34,130 & & & \\
\hline & 150,00 & 83,595 & & & \\
\hline Crude oil & 50,000 & 12,900 & $\begin{array}{l}\text { BA (by Bacillus } \\
\text { subtilis and } \\
\text { Acremonium sp) }\end{array}$ & 180 & (Ma et al., 2015) \\
\hline
\end{tabular}

Biostimulation and mineral fertilization=BIS-MIFE; Fertilization =FE; Bioaugmentation= BA; Phytoremedatio $={ }^{\wedge}$ PHYTO, * Concentration of HCs permitted by the Mexican regulation number NOM-138-SEMARNAT/SSA1-2012.

\section{Conclusion}

The strategy to follow for the remediation of a soil polluted with HCs depends upon the concentration and chemical nature of the pollutants. If the concentration of HCs is very high, 


\section{Macrothink}

physical removal, followed by chemical and biological treatments are strongly recommended. Furthermore, during the biological treatment, phytoremediation, or simultaneous bioremediation-phytoremediation and the use of PGPM are also highly advisable. The quantitative and qualitative analysis of soil to measure the HCs levels and remediation should always be complemented with bio- indicator tests. To unify criteria related to pollution of the environment, it is urgent to define international environmental regulations to be followed by all countries.

\section{Acknowledgement}

The authors thank CIC-Universidad Michoacana de San Nicolas de Hidalgo, under Research Project 2.7 (2018) and to BIONUTRA, SA de CV, Maravatio, Mich, México, for the financial support.

\section{References}

Abdulsalam, S., \& Omale A. B. (2009). Comparison of biostimulation and bioaugmentation techniques for the remediation of used motor oil contaminated soil. Brazilian Arch. Biol. Technol, 52, 747-754. https://doi.org/10.1590/S1516-89132009000300027

Abioye O. P., Agamuthu, P., \& Abdul Aziz, A. R. (2012). Biodegradation of used motor oil in soil using organic waste amendments. Biotechnol. Res. Int. https://doi.org/10.1155/2012/587041

Abioye, O. P., Agamuthu, P., \& Abdul Aziz, A. R. (2012). Phytotreatment of soil contaminated with used lubricating oil using Hibiscus cannabinus. Biodegradation, 23, 277-286. https://doi.org/10.1007/s10532-011-9506-9

Adenipekun \& Oyinkansola, C. (2008). Bioremediation of engine-oil polluted soil by Pleurotus tuber-regium Singer, a Nigerian white-rot fungus. African J. Biotechnol, 7, 055-058. https://doi.org/10.5897/AJB2008.000-5012

Agamuthu P., Abioye, O. P., \& Aziz, A. (2010). Phytoremediation of soil contaminated with used lubricating oil using Jatropha curcas. J. Hazard. Mater, 179, 891-894. https://doi.org/10.1016/j.jhazmat.2010.03.088

Agarry, S., \& Latinwo, G. (2015). Biodegradation of diesel oil in soil and its enhancement by application of bioventing and amendment with brewery waste effluents as Biostimulation-Bioaugmentation Agents. J. Ecol. Eng, 16, 82-91. https://doi.org/10.12911/22998993/1861

Almansoory, A. F., Idris, M., Abdullah, S. R. S, \& Anuar, N. (2014). Plant-microbe interaction of Serratia marcescens and Scirpus mucronatus on phytoremediation of gasoline contaminated soil. Int. J. ChemTech Res, 6, 556-564.

Asiabadi, F., Mirbagheri, S., Najafi, P., \& Moatar, F. (2014). Phytoremediation of petroleum-contaminated soils around Isfahan oil refinery (Iran) by sorghum and barley. Curr. World Environ, 9, 65-72. https://doi.org/10.12944/CWE.9.1.10 
Asquith, E. A., Geary, P. M., Nolan, A. L., \& Evans, C. A. (2012). Comparative Bioremediation of Petroleum Hydrocarbon-Contaminated Soil by Biostimulation, Bioaugmentation and Surfactant Addition. J. Environ. Sci. Eng, 1, 637-650.

Bahadure, S., Kalia, R., \& Chavan, R. (2013). Comparative Study of Bioremediation of Hydrocarbon Fuels. Int. J. Biotechnol. Bioeng. Res, 4, 677-686.

Basumatary, B., Saikia, R., \& Bordoloi, S. (2012). Phytoremediation of crude oil contaminated soil using nut grass, Cyperus rotundus. J Env. Biol, 33, 891-896.

Benyahia, F., Abdulkarim, M., Zekri, A., Chaalal, O., \& Hasanain, H. (2005). Bioremediation of crude oil contaminated soils: A black art or an engineering challenge? Process Saf. Environ. Prot, 83, 364-370. https://doi.org/10.1205/psep.04388

Burghal, A. A., Al-Mudaffar, N. A., \& Mahdi, K. H. (2015). Ex situ bioremediation of soil contaminated with crude oil by use of actinomycetes consortia for process bioaugmentation. Eur. J. Exp. Biol, 5, 24-30.

Cai, Z., Zhou, Q., Peng, S., \& Li, K. (2010). Promoted biodegradation and microbiological effects of petroleum hydrocarbons by Impatiens balsamina L. with strong endurance. $J$. Hazard. Mater, 183, 731-7. https://doi.org/10.1016/j.jhazmat.2010.07.087

Chuluun, B., Shah, S. H., \& Rhee, J. (2014). Bioaugmented Phytoremediation : A Strategy for Reclamation of Diesel Oil-contaminated Soils. Int. J. Agric. Biol, 16, 624-628.

Diab, E. A. (2008). Phytoremediation of Oil Contaminated Desert Soil Using the Rhizosphere Effects. Glob. J. Environ. Res, 2, 66-73.

Escalante-Espinosa, E., Gallegos-Martínez, M. E., Favela-Torres, E., \& Gutiérrez-Rojas, M. (2005). Improvement of the hydrocarbon phytoremediation rate by Cyperus laxus Lam. inoculated with a microbial consortium in a model system. Chemosphere, 59, 405-413. https://doi.org/10.1016/j.chemosphere.2004.10.034

Gerhardt, K. E., Huang, X. D., Glick, B. R., \& Greenberg, B. M. (2009). Phytoremediation and rhizoremediation of organic soil contaminants: Potential and challenges. Plant Sci, 176, 20-30. https://doi.org/10.1016/j.plantsci.2008.09.014

Haritash, A. K., \& Kaushik, C. P. (2009). Biodegradation aspects of Polycyclic Aromatic Hydrocarbons (PAHs): A review. J. Hazard. Mater; 169, 1-15. https://doi.org/10.1016/j.jhazmat.2009.03.137

Hernández-Espriú, A., Sánchez-León, E., Martínez-Santos, P., \& Torres, L. (2013). Remediation of a diesel-contaminated soil from a pipeline accidental spill: enhanced biodegradation and soil washing processes using natural gums and surfactants. J. Soils Sediments, 13, 152-165. https://doi.org/10.1007/s11368-012-0599-5

Johnsen, A. R., Wick, L. Y., \& Harms, H. (2005). Principles of microbial PAH-degradation in soil. Environ. Pollut, 133, 71-84. https://doi.org/10.1016/j.envpol.2004.04.015

Juhasz, A. L., \& Naidu, R. (2000). Bioremediation of high molecular weight polycyclic 
aromatic hydrocarbons: a review of the microbial degradation of benzo [a] pyrene. Int. Biodeterior. Biodegradation, 45, 57-88. https://doi.org/10.1016/S0964-8305(00)00052-4

Kumar, A., Bisht, B., Joshi, V., \& Dhewa, T. (2011). Review on Bioremediation of Polluted Environment : A Management Tool. Int. J. Environ. Sci, 1, 1079-1093.

Lee, S.-H., Oh, B.-I., \& Kim, J. (2008). Effect of various amendments on heavy mineral oil bioremediation and soil microbial activity. Bioresour. Technol, 99, 2578-2587. https://doi.org/10.1016/j.biortech.2007.04.039

Ma, X.-K., Ding, N., \& Peterson, E. (2015). Bioaugmentation of soil contaminated with high-level crude oil through inoculation with mixed cultures including Acremonium sp., Biodegradation, 26, 259-269. https://doi.org/10.1007/s10532-015-9732-7

Mancera-López, M. E., Esparza-García, F., Chávez-Gómez, B., Rodríguez-Vázquez, R., Saucedo-Castañeda, G., \& Barrera-Cortés J. (2008). Bioremediation of an aged hydrocarbon-contaminated soil by a combined system of biostimulation-bioaugmentation with filamentous fungi. Int. Biodeterior. Biodegradation, 61, 151-160. https://doi.org/10.1016/j.ibiod.2007.05.012

Merkl, N., Schultze-Kraft, R., \& Infante, C. (2005). Phytoremediation in the tropics Influence of heavy crude oil on root morphological characteristics of graminoids. Environ. Pollut, 138, 86-91. https://doi.org/10.1016/j.envpol.2005.02.023

Millioli, V. S., Servulo, E. L. C., Sobral, L. G. S., \& De Carvalho, D. D. (2009). Bioremediation of crude oil-bearing soil: evaluating the effect of rhamnolipid addition to soil toxicity and to crude oil biodegradation efficiency. Glob. NEST J, 11, 181-188.

Minai-Tehrani, D., Tameh A. T., Rashidfarokhi, A., Noormohammadi, A., Khodakarami, A., \& Taleb, M. (2012). The Effect of Light Crude Oil-Contaminated Soil on the Growth and Germination of Sorghum bicolor. Eur. J. Plant Sci. Biotechnol, 6, 81-84.

Minoui, S., Minai-Tehrani, D., \& Shahriari, M. H. (2015). Phytoremediation of Crude Oil-Contaminated Soil by Medicago sativa (Alfalfa) and the Effect of Oil on its Growth. In M. Öztürk, M. Ashraf, A. Aksoy, \& M. S. A. Ahmad (Eds.), Phytoremediation Green Energy (pp. 123-129). SE- 8, Springer Netherlands.

Mishra, S., Jyot, J., Kuhad, R. C., \& Lal, B. (2001). Evaluation of Inoculum Addition to Stimulate in Situ Bioremediation of Oily-Sludge-Contaminated Soil. Appl. Environ. Microbiol, 67, 1675-1681. https://doi.org/10.1128/AEM.67.4.1675-1681.2001

Najirad, S., Alikhani, H. A., Hashemi, M. M., Naghavi, M. R., \& Ghavidel, A. (2012). Hydrocarbon bioremediation efficiency by two indigenous bacterial strains in contaminated soils. World Appl. Sci. J, 17, 792-796.

Njoku, O. B., \& Akinola, M. (2009). Phytoremediation of crude oil contaminated soil the effect of growth of Glycine max on the physico-chemistry and crude oil contents of soil. Nat. Sci, 7, 79-87. 
NOM-138-SEMARNAT Límites máximos permisibles hidrocarburos en suelo y especificaciones caracterización y remediación, n.d. http://www.dof.gob.mx/ nota_detalle.php?codigo $=5313544 \&$ fecha $=10 / 09 / 2013$.

Nwoko, C. O. (2010). Trends in phytoremediation of toxic elemental and organic pollutants. African J. Biotechnol, 9, 6010-6016.

Ohio EPA, Guidance for Assessing Petroleum Hydrocarbons in Soil, 04 (2010) 1-15.

Onuoha, S. C. (2013). Stimulated Biodegradation of Spent Lubricating Motor oil in Soil Amended with Animal droppings. J. of Natural Sci. Research, 3, 106-117.

Ozawa, S., Ikeura, H., Kaimi, E., \& Tamaki, M. (2015). Selection of the Most Effective Cultivar of Genus Zinnia Flowers for Phytoremediation of Oil-contaminated Soil. Int. J. Plant Soil Sci, 4, 61-71. https://doi.org/10.9734/IJPSS/2015/13035

Peng, S., Zhou, Q., Cai, Z., \& Zhang, Z. (2009). Phytoremediation of petroleum contaminated soils by Mirabilis Jalapa L. in a greenhouse plot experiment. J. Hazard. Mater, 168, 1490-1496. https://doi.org/10.1016/j.jhazmat.2009.03.036

Romanus, A. A., Ikechukwu, E. F., Patrick, A. S., Goddey, U., \& Helen, O. (2015). Efficiency of Plantain Peels and Guinea Corn Shaft for Bioremediation of Crude Oil Polluted Soil. $J$. Microbiol. Res, 5, 31-40.

Sharma, S. (2012). Bioremediation: Features, Strategies and applications. Asian J. Pharm. Life Sci, 2, 202-213.

Shirdam, R., Zand, A. D., Bidhendi, G. N., \& Mehrdadi, N. (2008). Phytoremediation of hydrocarbon-contaminated soils with emphasis on the effect of petroleum hydrocarbons on the growth of plant species. Phytoprotection, 89, 21-29. https://doi.org/10.7202/000379ar

Tanee, L. A., \& Akonye, F. B. G. (2009). Effectiveness of Vigna Unguiculata as a Phytoremediation Plant in the remediation of Crude Oil polluted soil for Cassava (Manihot Esculenta; Crantz) Cultivation. J. Appl. Sci. Environ. Manag, 13, 43-47.

Tang, J., Wang, R., Niu, X., \& Zhou, Q. (2010). Enhancement of soil petroleum remediation by using a combination of ryegrass (Lolium perenne) and different microorganisms. Soil Tillage Res, 110, 87-93. https://doi.org/10.1016/j.still.2010.06.010

Tang, J. C., Wang, R. G., Niu, X. W., Wang, M., Chu, H. R, \& Zhou, Q. X. (2010). Characterisation of the rhizoremediation of petroleum-contaminated soil: effect of different influencing factors. Biogeosciences, 7, 3961-3969. https://doi.org/10.5194/bg-7-3961-2010

Thapa, B., Kc, A. K., \& Ghimire, A. (2012). A Review on Bioremediation of Petroleum Hydrocarbon Contaminants In Soil, Kathmandu Univ. J. Sci. Eng. Teichnol, 8, 164-170. https://doi.org/10.3126/kuset.v8i1.6056

Toledo Ramos, D., Maranho, L., Godoi, A., Carvalho Filho, M. daSilva, Lacerda, L., de Vasconcelos, E. (2009). Petroleum Hydrocarbons Rhizodegradation by Sebastiania commersoniana (Baill.) L. B. S M. \& Downs. Water, Air, Soil Pollut. Focus, 9, 293-302. 
https://doi.org/10.1007/s11267-009-9208-z

Vallejo, V., Salgado, L., \& Roldan, F. (2005). Evaluación de la bioestimulación en la biodegradación de TPHs en suelos contaminados con petróleo. Rev. Colomb. Biotecnol, 2, 67-78.

Zand, A. D., Bidhendi, G. N., \& Mehrdadi, N. (2010). Phytoremediation of total petroleum hydrocarbons (TPHs) using plant species in Iran, Turkish. J. Agric. For, 34, 429-438. Zhang, Z., Zhou, Q., Peng, S., \& Cai Z. (2010). Remediation of petroleum contaminated soils by joint action of Pharbitis nil L. and its microbial community. Sci. Total Environ, 408, $5600-5605$.

\section{Copyright Disclaimer}

Copyright reserved by the author(s).

This article is an open-access article distributed under the terms and conditions of the Creative Commons Attribution license (http://creativecommons.org/licenses/by/3.0/). 